\title{
ON HILBERT BOUNDARY VALUE PROBLEM FOR BELTRAMI EQUATION
}

\author{
Vladimir Gutlyanskii, Vladimir Ryazanov, \\ Eduard Yakubov and Artyem Yefimushkin
} National Academy of Sciences of Ukraine, Institute of Applied Mathematics and Mechanics
Generala Batyuka str. 19, 84116 Slavyansk, Ukraine; vgutlyanskii@gmail.com

National University of Cherkasy, Physics Department, Laboratory of Mathematical Physics and National Academy of Sciences of Ukraine, Institute of Applied Mathematics and Mechanics Generala Batyuka str. 19, 84116 Slavyansk, Ukraine; Ryazanov@nas.gov.ua

Holon Institute of Technology

Golomb St. 52, Holon, 5810201, Israel; yakubov@hit.ac.il

National Academy of Sciences of Ukraine, Institute of Applied Mathematics and Mechanics Generala Batyuka str. 19, 84116 Slavyansk, Ukraine; a.yefimushkin@gmail.com

In memory of Professor Bogdan Bojarski

\begin{abstract}
We study the Hilbert boundary value problem for the Beltrami equation in the Jordan domains satisfying the quasihyperbolic boundary condition by Gehring-Martio, generally speaking, without $(A)$-condition by Ladyzhenskaya-Ural'tseva that was standard for boundary value problems in the PDE theory. Assuming that the coefficients of the problem are functions of countable bounded variation and the boundary data are measurable with respect to the logarithmic capacity, we prove the existence of the generalized regular solutions. As a consequence, we derive the existence of nonclassical solutions of the Dirichlet, Neumann and Poincaré boundary value problems for generalizations of the Laplace equation in anisotropic and inhomogeneous media.
\end{abstract}

\section{Introduction}

Hilbert [31] studied the boundary value problem formulated as follows: To find an analytic function $f(z)$ in a domain $D$ bounded by a rectifiable Jordan contour $C$ that satisfies the boundary condition

$$
\lim _{z \rightarrow \zeta} \operatorname{Re}\{\overline{\lambda(\zeta)} f(z)\}=\varphi(\zeta) \quad \forall \zeta \in C,
$$

where both the coefficient $\lambda$ and the boundary date $\varphi$ of the problem are continuously differentiable with respect to the natural parameter $s$ on $C$.

Moreover, it was assumed by Hilbert that $\lambda \neq 0$ everywhere on $C$. The latter allows us, without loss of generality, to consider that $|\lambda| \equiv 1$ on $C$. Note that the quantity $\operatorname{Re}\{\bar{\lambda} f\}$ in (1.1) means a projection of $f$ into the direction $\lambda$ interpreted as vectors in $\mathbf{R}^{2}$.

The reader can find a rather comprehensive treatment of the theory in the new excellent books $[10,11,29,45]$. We also recommend to make familiar with the historic

https://doi.org/10.5186/aasfm.2020.4552

2010 Mathematics Subject Classification: Primary 30C062, 31A05, 31A20, 31A25, 31B25, 35J61; Secondary 30E25, 31C05, 34M50, 35F45, 35Q15.

Key words: Hilbert boundary value problem, Beltrami equation, quasihyperbolic boundary condition, logarithmic capacity, angular limits. 
surveys contained in the monographs $[21,41,55]$ on the topic with an exhaustive bibliography and take a look at our recent papers [26, 28, 47].

In this paper we study the Hilbert boundary value problem in a wider class of functions than those of analytic. Namely, instead of analytic functions we will consider quasiconformal functions $F$ represented as a composition of analytic functions $\mathcal{A}$ and quasiconformal mappings $f$, see [38, Chapter VI]. In this connection, we need to recall some definitions and notations from the theory of quasiconformal mappings in the plane.

Let $D$ be a domain in the complex plane $\mathbf{C}$ and let $\mu: D \rightarrow \mathbf{C}$ be a measurable function with $|\mu(z)|<1$ a.e. The equation of the form

$$
f_{\bar{z}}=\mu(z) f_{z}
$$

where $f_{\bar{z}}=\bar{\partial} f=\left(f_{x}+i f_{y}\right) / 2, f_{z}=\partial f=\left(f_{x}-i f_{y}\right) / 2, z=x+i y, f_{x}$ and $f_{y}$ are partial derivatives of the function $f$ in $x$ and $y$, respectively, is said to be a Beltrami equation. The equation (1.2) is said to be nondegenerate if $\|\mu\|_{\infty}<1$, see e.g. [3], [14] and [38], that we will assume later on.

Homeomorphic solutions $f$ of a nondegenerate equation (1.2) in the class $W_{\text {loc }}^{1,2}$ are called quasiconformal mappings. It is easy to see that every quasiconformal function $F=\mathcal{A} \circ f$ satisfies the same Beltrami equation as $f$.

Recall also that the images of the unit disk $\mathbf{D}=\{z \in \mathbf{C}:|z|<1\}$ under the quasiconformal mappings of $\mathbf{C}$ onto itself are called quasidisks and their boundaries are called quasicircles or quasiconformal curves. It is known that every smooth (or Lipschitz) Jordan curve is a quasiconformal curve and, at the same time, quasiconformal curves can be locally nonrectifiable as it follows from the known examples, see e.g. the point II.8.10 in [38]. On the other hand, see Section 3, quasicircles satisfy the well-known $(A)$-condition, which is standard in the theory of boundary value problems for PDE, see e.g. [36].

Proceeding from the above, the problem under consideration is to find the quasiconformal function, satisfying both the Beltrami equation (1.2) in a Jordan domain $D$ and the Hilbert boundary condition (1.1). We substantially weaken the regularity conditions both on the functions $\lambda$ and $\varphi$ in the boundary condition (1.1) and on the boundary $C$ of the domain $D$. On the one hand, we will deal with the coefficients $\lambda$ of countable bounded variation and the boundary data $\varphi$ which are measurable with respect to the logarithmic capacity. On the other hand, the fundamental BeckerPommerenke result in [9] allows us to study the Hilbert boundary value problem in domains $D$ with the quasihyperbolic boundary condition introduced in [23], see also [7]. It is important to note that such domains may fail to satisfy the $(A)$-condition, see Section 3.

Let $D$ be a Jordan domain such that it has a tangent at a point $\zeta \in \partial D$. A path in $D$ terminating at $\zeta$ is called nontangential if its part in a neighborhood of $\zeta$ lies inside of an angle in $D$ with the vertex at $\zeta$. The limit along all nontangential paths at $\zeta$ is called angular at the point. The latter notion is a standard tool for the study of the boundary behavior of analytic and harmonic functions, see e.g. [17, 34, 46]. Further, the Hilbert boundary condition (1.1) will be understood precisely in the sense of the angular limit.

The notion of the logarithmic capacity is the important tool for our research, see e.g. $[15,42,43]$, because the sets of zero logarithmic capacity are transformed under quasiconformal mappings into the sets of zero logarithmic capacity. Note that, as it follows from the classic Ahlfors-Beurling example, see [4], the sets of zero length 
as well as the sets of zero harmonic measure are not invariant under quasiconformal mappings.

Dealing with measurable boundary date functions $\varphi(\zeta)$ with respect to the logarithmic capacity, we will use the abbreviation q.e. (quasi-everywhere) on a set $E \subset \mathbf{C}$, if a property holds for all $\zeta \in E$ except its subset of zero logarithmic capacity, see [37].

\section{Definitions and preliminary remarks}

Given a bounded Borel set $E$ in the plane $\mathbf{C}$, a mass distribution on $E$ is a nonnegative completely additive function $\nu$ of a set defined on its Borel subsets with $\nu(E)=1$. The function

$$
U^{\nu}(z):=\int_{E} \log \left|\frac{1}{z-\zeta}\right| d \nu(\zeta)
$$

is called a logarithmic potential of the mass distribution $\nu$ at a point $z \in \mathbf{C}$. A logarithmic capacity $C(E)$ of the Borel set $E$ is the quantity

$$
C(E)=e^{-V}, \quad V=\inf _{\nu} V_{\nu}(E), \quad V_{\nu}(E)=\sup _{z} U^{\nu}(z) .
$$

It is also well-known the following geometric characterization of the logarithmic capacity, see e.g. the point 110 in [42]:

$$
C(E)=\tau(E):=\lim _{n \rightarrow \infty} V_{n}^{\frac{2}{n(n-1)}}
$$

where $V_{n}$ denotes the supremum of the product

$$
V\left(z_{1}, \ldots, z_{n}\right)=\prod_{k<l}^{l=1, \ldots, n}\left|z_{k}-z_{l}\right|
$$

taken over all collections of points $z_{1}, \ldots, z_{n}$ in the set $E$. Following Fékete, see [20], the quantity $\tau(E)$ is called the transfinite diameter of the set $E$.

Remark 2.1. Thus, we see that if $C(E)=0$, then $C(f(E))=0$ for an arbitrary mapping $f$ that is continuous by Hölder and, in particular, for quasiconformal mappings on compact sets, see e.g. Theorem II.4.3 in [38].

In order to introduce sets that are measurable with respect to logarithmic capacity, we define, following [15], inner $C_{*}$ and outer $C^{*}$ capacities:

$$
C_{*}(E):=\sup _{F \subseteq E} C(E), \quad C^{*}(E):=\inf _{E \subseteq O} C(O),
$$

where supremum is taken over all compact sets $F \subset \mathbf{C}$ and infimum is taken over all open sets $O \subset \mathbf{C}$. A set $E \subset \mathbf{C}$ is called measurable with respect to the logarithmic capacity if $C^{*}(E)=C_{*}(E)$, and the common value of $C_{*}(E)$ and $C^{*}(E)$ is still denoted by $C(E)$.

A function $\varphi: E \rightarrow \mathbf{C}$ defined on a bounded set $E \subset \mathbf{C}$ is called measurable with respect to logarithmic capacity if, for all open sets $O \subseteq \mathbf{C}$, the sets

$$
\Omega=\{z \in E: \varphi(z) \in O\}
$$

are measurable with respect to logarithmic capacity. It is clear from the definition that the set $\mathrm{E}$ is itself measurable with respect to logarithmic capacity.

Note also that sets of logarithmic capacity zero coincide with sets of the so-called absolute harmonic measure zero introduced by Nevanlinna, see [42, Chapter V]. Hence a set $E$ is of (Hausdorff) length zero if $C(E)=0$, see [42, Theorem V.6.2]. However, 
there exist sets of length zero having a positive logarithmic capacity, see e.g. [15, Theorem IV.5].

Remark 2.2. It is known that Borel sets and, in particular, compact and open sets are measurable with respect to logarithmic capacity, see e.g. [15, Lemma I.1 and Theorem III.7]. Moreover, as it follows from the definition, any set $E \subset \mathbf{C}$ of finite logarithmic capacity can be represented as a union of a sigma-compactum (union of countable collection of compact sets) and a set of logarithmic capacity zero. It is also known that the Borel sets and, in particular, compact sets are measurable with respect to all Hausdorff's measures and, in particular, with respect to measure of length, see e.g. [52, Theorem II(7.4)]. Consequently, any set $E \subset \mathbf{C}$ of finite logarithmic capacity is measurable with respect to measure of length. Thus, on such a set any function $\varphi: E \rightarrow \mathbf{C}$ being measurable with respect to logarithmic capacity is also measurable with respect to measure of length on $E$. However, there exist functions that are measurable with respect to measure of length but not measurable with respect to logarithmic capacity, see e.g. [15, Theorem IV.5].

We call $\lambda: \partial \mathbf{D} \rightarrow \mathbf{C}$ a function of bounded variation, write $\lambda \in \mathcal{B V}(\partial \mathbf{D})$, if

$$
V_{\lambda}(\partial \mathbf{D}):=\sup \sum_{j=1}^{j=k}\left|\lambda\left(\zeta_{j+1}\right)-\lambda\left(\zeta_{j}\right)\right|<\infty,
$$

where the supremum is taken over all finite collections of points $\zeta_{j} \in \partial \mathbf{D}, j=1, \ldots, k$, with the cyclic order meaning that $\zeta_{j}$ lies between $\zeta_{j+1}$ and $\zeta_{j-1}$ for every $j=1, \ldots, k$. Here we assume that $\zeta_{k+1}=\zeta_{1}=\zeta_{0}$. The quantity $V_{\lambda}(\partial \mathbf{D})$ is called the variation of the function $\lambda$.

Remark 2.3. It is clear by the triangle inequality that if we add new intermediate points in the collection $\zeta_{j}, j=1, \ldots, k$, then the sum in (2.7) does not decrease. Thus, the given supremum is attained as $\delta=\sup _{j=1, \ldots k}\left|\zeta_{j+1}-\zeta_{j}\right| \rightarrow 0$. Note also that by the definition $V_{\lambda}(\partial \mathbf{D})=V_{\lambda \circ h}(\partial \mathbf{D})$, i.e., the variation is invariant under every homeomorphism $h: \partial \mathbf{D} \rightarrow \partial \mathbf{D}$ and, thus, the definition can be extended in a natural way to an arbitrary Jordan curve in $\mathbf{C}$.

The following statement was proved as Proposition 5.1 in the paper [18] where the function $\alpha_{\lambda}$ has been called by a function of argument of $\lambda$.

Proposition 2.4. For every function $\lambda: \partial \mathbf{D} \rightarrow \partial \mathbf{D}$ of the class $\mathcal{B V}(\partial \mathbf{D})$ there is a function $\alpha_{\lambda}: \partial \mathbf{D} \rightarrow \mathbf{R}$ of the class $\mathcal{B V}(\partial \mathbf{D})$ with $V_{\alpha_{\lambda}} \leq V_{\lambda} 3 \pi / 2$ such that $\lambda(\zeta)=\exp \left\{i \alpha_{\lambda}(\zeta)\right\}$ for all $\zeta \in \partial \mathbf{D}$.

Now, we call $\lambda: \partial \mathbf{D} \rightarrow \mathbf{C}$ a function of countable bounded variation, write $\lambda \in$ $\mathcal{C B V}(\partial \mathbf{D})$, if there is a countable collection of mutually disjoint $\operatorname{arcs} \gamma_{n}$ of $\partial \mathbf{D}, n=$ $1,2, \ldots$, on each of which the restriction of $\lambda$ is of bounded variation $V_{n}$ and the set $\partial \mathbf{D} \backslash \bigcup \gamma_{n}$ has logarithmic capacity zero. In particular, the latter holds true if $\partial \mathbf{D} \backslash \bigcup \gamma_{n}$ is countable. Choosing smaller $\gamma_{n}$, we may assume that $\sup _{n} V_{n}<\infty$. It is clear, such functions can be singular enough, see e.g. [16].

The definition is also extended in the natural way to an arbitrary Jordan curve $\Gamma$ in $\mathbf{C}$. Later on, $L_{c}^{\infty}(\Gamma)$ denotes the class of all functions $\alpha: \Gamma \rightarrow \mathbf{R}$ which are measurable with respect to logarithmic capacity such that $\alpha$ is q.e. bounded on $\Gamma$.

Proposition 2.5. For every function $\lambda: \partial \mathbf{D} \rightarrow \partial \mathbf{D}$ in the class $\mathcal{C B V}(\partial \mathbf{D})$ there is a function $\alpha_{\lambda}: \partial \mathbf{D} \rightarrow \mathbf{R}$ in the class $L_{c}^{\infty}(\partial \mathbf{D}) \cap \mathcal{C B V}(\partial \mathbf{D})$ such that

$$
\lambda(\zeta)=\exp \left\{i \alpha_{\lambda}(\zeta)\right\} \quad \text { q.e. on } \partial \mathbf{D} \text {. }
$$


Proof. Denote by $\lambda_{n}$ the function on $\partial \mathbf{D}$ that is equal to $\lambda$ on $\gamma_{n}$ and to 1 outside of $\gamma_{n}$. Let $\alpha_{n}$ correspond to $\lambda_{n}$ by Proposition 2.4. Then its variation $V_{n}^{*} \leq V_{n} 3 \pi / 2$. With no loss of generality we may assume that $\alpha_{n} \equiv 0$ outside of $\gamma_{n}$. Set $\alpha=\sum_{n=1}^{\infty} \alpha_{n}$. Then $\alpha \in \mathcal{C B} \mathcal{V}(\partial \mathbf{D})$ and $\lambda(\zeta)=\exp \{i \alpha(\zeta)\}$ q.e. on $\partial \mathbf{D}$. Applying the corresponding shifts (divisible $2 \pi$ ), we may change $\alpha_{n}$ on $\gamma_{n}$ through $\alpha_{n}^{*}$ with $\left|\alpha_{n}^{*}\right| \leq \pi$ at the middle point of $\gamma_{n}$. Then it is clear that the new function $\alpha^{*} \in \mathcal{C B V}(\partial \mathbf{D})$ and $\lambda(\zeta)=\exp \left\{i \alpha^{*}(\zeta)\right\}$ q.e. on $\partial \mathbf{D}$ and, moreover, $\left|\alpha^{*}\right| \leq \pi+V_{n} 3 \pi / 2$ on every $\gamma_{n}$, i.e. $\left|\alpha^{*}\right|$ is bounded on the set $\partial \mathbf{D} \backslash \bigcup \gamma_{n}$. In addition, by the construction, the function $\alpha^{*}$ is continuous q.e. on $\partial \mathbf{D}$. Hence $\alpha^{*} \in L_{c}^{\infty}(\partial \mathbf{D})$.

We say that a Jordan curve $\Gamma$ in $\mathbf{C}$ is almost smooth if $\Gamma$ has a tangent quasieverywhere. Here we say that a straight line $L$ in $\mathbf{C}$ is tangent to $\Gamma$ at a point $z_{0} \in \Gamma$ if

$$
\limsup _{z \rightarrow z_{0}, z \in \Gamma} \frac{\operatorname{dist}(z, L)}{\left|z-z_{0}\right|}=0 .
$$

In particular, $\Gamma$ is almost smooth if $\Gamma$ has a tangent at all its points except a countable set. The nature of such Jordan curves $\Gamma$ is complicated enough because the countable set can be everywhere dense in $\Gamma$.

Remark 2.6. By Corollary of Theorem 1 in [9], a conformal mapping of a Jordan domain $D$ in $\mathbf{C}$ with the quasihyperbolic boundary condition, see the definition in Section 3, onto the unit disk $\mathbf{D}$, as well as its inverse are Hölder continuous in the closure of $D$ and $\mathbf{D}$, respectively. Thus, by Remark 2.1 these mappings keep the sets of the logarithmic capacity zero on boundaries of $D$ and D. Consequently, by Remark 2.2, such mappings also keep boundary functions which are measurable with respect to the logarithmic capacity. These facts are key for the research of the boundary value problems in the given domains.

\section{On domains with quasihyperbolic boundary condition}

Let $D$ be a domain in $\mathbf{C}$. As usual, here $k_{D}\left(z, z_{0}\right)$ denotes the quasihyperbolic distance between points $z$ and $z_{0}$ in $D$,

$$
k_{D}\left(z, z_{0}\right):=\inf _{\gamma} \int_{\gamma} \frac{d s}{d(\zeta, \partial D)},
$$

introduced in the paper [24], see also the monographs [5] and [56]. Here $d(\zeta, \partial D)$ denotes the Euclidean distance from the point $\zeta \in D$ to $\partial D$ and the infimum is taken over all rectifiable curves $\gamma$ joining the points $z$ and $z_{0}$ in $D$. if

Further, it is said that a domain $D$ satisfies the quasihyperbolic boundary condition

$$
k_{D}\left(z, z_{0}\right) \leq a \ln \frac{d\left(z_{0}, \partial D\right)}{d(z, \partial D)}+b \quad \forall z \in D
$$

for constants $a$ and $b$ and a point $z_{0} \in D$. The latter notion was introduced in [23] but, before it, was first applied in [9].

Remark 3.1. Quasidisks $D$ satisfy the quasihyperbolic boundary condition. Indeed, as well-known the Riemann conformal mapping $\omega: D \rightarrow \mathbf{D}$ is extended to a quasiconformal mapping of $\mathbf{C}$ onto itself, see e.g. [38, Theorem II.8.3]. By one of the main Bojarski results, see [12] and [13], Theorem 3.5, the derivatives of quasiconformal mappings in the plane are locally integrable with some power $q>2$. Note also that its Jacobian $J(w)=\left|\omega_{w}\right|^{2}-\left|\omega_{\bar{w}}\right|^{2}$, see e.g. [3, I.A(9)]. Consequently, in this case 
$J \in L^{p}(D)$ for some $p>1$ and we have the desired conclusion by the criterion in $[7$, Theorem 2.4].

Recall that a domain $D$ in $\mathbf{R}^{n}, n \geq 2$, is called satisfying $(A)$-condition if

$$
\text { mes } D \cap B(\zeta, \rho) \leq \Theta_{0} \operatorname{mes} B(\zeta, \rho) \quad \forall \zeta \in \partial D, \rho \leq \rho_{0}
$$

for some $\Theta_{0}$ and $\rho_{0} \in(0,1)$, see [36, 1.1.3]. Recall also that a domain $D$ in $\mathbf{R}^{n}$, $n \geq 2$, is said to be satisfying the outer cone condition if there is a cone that makes possible to be touched by its top to every boundary point of $D$ from the completion of $D$ after its suitable rotations and shifts. It is clear that the outer cone condition implies $(A)$-condition. It is well known that the above conditions are standard in the theory of boundary value problems for the partial differential equations.

Remark 3.2. Note that quasidisks $D$ satisfy $(A)$-condition. Indeed, the quasidisks are the so-called $Q E D$-domains by Gehring-Martio, see [22, Theorem 2.22], and the latter satisfy the condition

$$
\text { mes } D \cap B(\zeta, \rho) \geq \Theta_{*} \operatorname{mes} B(\zeta, \rho) \quad \forall \zeta \in \partial D, \rho \leq \operatorname{diam} D,
$$

for some $\Theta_{*} \in(0,1)$, see [22, Lemma 2.13], and quasidisks (as domains with quasihyperbolic boundary condition) have boundaries of the Lebesgue measure zero, see e.g. [7, Theorem 2.4]. Thus, it remains to note that, by definition, the completions of quasidisks $D$ in the the extended complex plane $\overline{\mathbf{C}}:=\mathbf{C} \cup\{\infty\}$ are also quasidisks up to the inversion with respect to a circle in $D$.

As we know, the first example of a simply connected plane domain $D$ with the quasihyperbolic boundary condition which is not a quasidisk was constructed in $[9$, Theorem 2]. However, this domain had $(A)$-condition.

Remark 3.3. Probably one of the simplest examples of a domain $D$ with the quasihyperbolic boundary condition and without $(A)$-condition is the union of 3 open disks with the radius 1 centered at the points 0 and $1 \pm i$. It is clear that the domain has zero interior angle at its boundary point 1 and by Remark 3.2 it is not a quasidisk. Note that $\partial D$ is almost smooth. Thus, there exist almost smooth Jordan curves with the quasihyperbolic boundary condition that are not quasiconformal curves.

From now on we will naturally assume that the boundary Jordan curves $\Gamma:=\partial D$ are almost smooth.

\section{Boundary correlation of conjugate harmonic functions}

It is known the very delicate observation due to Lusin that harmonic functions in the unit circle with continuous (even absolutely continuous !) boundary data can have conjugate harmonic functions whose boundary data are not continuous functions, furthemore, they can be even not essentially bounded in neighborhoods of each point of the unit circle, see e.g. [8, Theorem VIII.13.1]. Thus, a correlation between boundary data of conjugate harmonic functions is not a simple matter, see e.g. [34, I.E], see also [50] and [51].

The following statement was first proved for the case of bounded variation in [18]. Here we give an alternative proof of this significant fact and extend it to the case of countable bounded variation. 
Lemma 4.1. Let $\alpha: \partial \mathbf{D} \rightarrow \mathbf{R}$ be in the class $L_{c}^{\infty}(\partial \mathbf{D}) \cap \mathcal{C B V}(\partial \mathbf{D})$ and let $u: \mathbf{D} \rightarrow \mathbf{R}$ be a bounded harmonic function such that

$$
\lim _{z \rightarrow \zeta} u(z)=\alpha(\zeta)
$$

at every point of continuity of $\alpha$ and let $v$ be its conjugate harmonic function. Then $v$ has the angular limit

$$
\lim _{z \rightarrow \zeta} v(z)=\beta(\zeta) \quad \text { q.e. on } \partial \mathbf{D},
$$

where the function $\beta: \partial \mathbf{D} \rightarrow \mathbf{R}$ is measurable with respect to the logarithmic capacity.

Proof. Let us start from the case $\alpha \in \mathcal{B V}(\partial \mathbf{D})$. In this case $\alpha$ has at most a countable set $S$ of points of discontinuity and, consequently, $S$ is of zero logarithmic capacity. Hence by the generalized maximum principle, see e.g. the point 115 in [42], such a function $u$ is unique and, thus, $u$ can be represented as the Poisson integral of the function $\alpha$, see e.g. [34, Theorem I.D.2.2],

$$
u\left(r e^{i \vartheta}\right)=\frac{1}{2 \pi} \int_{-\pi}^{\pi} \frac{1-r^{2}}{1-2 r \cos (\vartheta-t)+r^{2}} \alpha\left(e^{i t}\right) d t .
$$

Here the Poisson kernel is a real part of the analytic function $(\zeta+z) /(\zeta-z), \zeta=e^{i t}$, $z=r e^{i \vartheta}$, and by the Weierstrass theorem, see e.g. [25, Theorem 1.1.1], the Schwartz integral

$$
f(z):=\frac{1}{2 \pi i} \int_{\partial \mathbf{D}} \alpha(\zeta) \frac{\zeta+z}{\zeta-z} \frac{d \zeta}{\zeta}
$$

gives the analytic function $f=u+i v$ in $\mathbf{D}$ with $u=\operatorname{Re} f, v=\operatorname{Im} f$, and

$$
f(z)=\frac{1}{2 \pi} \int_{-\pi}^{\pi} \alpha\left(e^{i t}\right) \frac{e^{i t}+z}{e^{i t}-z} d t=C+\frac{z}{\pi} \int_{-\pi}^{\pi} \frac{F(t)}{1-e^{-i t} z} d t
$$

where $F(t)=e^{-i t} \alpha\left(e^{i t}\right)$ and $C=\frac{1}{2 \pi} \int_{-\pi}^{\pi} \alpha\left(e^{i t}\right) d t$. By Theorem 2(c) in [54] the function $f(z)$ has angular limits $f(\zeta)$ as $z \rightarrow \zeta$ q.e. on $\partial \mathbf{D}$ because the function $F$ is of bounded variation. It remains to note that $f(\zeta)=\lim _{n \rightarrow \infty} f_{n}(\zeta)$, where $f_{n}(\zeta)=f\left(r_{n} \zeta\right)$, for an arbitrary sequence $r_{n} \rightarrow 1-0$ as $n \rightarrow \infty$ q.e. on $\partial \mathbf{D}$ and, thus, $f(\zeta)$ is measurable with respect to logarithmic capacity because the functions $f_{n}(\zeta)$ are so as continuous functions on $\partial \mathbf{D}$, see e.g. [19, 2.3.10].

Now, let $\alpha \in \mathcal{C B V}(\partial \mathbf{D})$. Then its set of points of discontinuity is at most of zero logarithmic capacity. Hence again by the generalized maximum principle the bounded function $u$ satisfying (4.1) is unique. Moreover, $\alpha \in L_{c}^{\infty}(\partial \mathbf{D})$ and, consequently, $u$ can be represented by the Poisson integral (4.3) and the Schwartz integral (4.4) gives the analytic function $f=u+i v$ in $\mathbf{D}$, where

$$
v\left(r e^{i \vartheta}\right)=\frac{1}{2 \pi} \int_{-\pi}^{\pi} \frac{2 r \sin (\vartheta-t)}{1-2 r \cos (\vartheta-t)+r^{2}} \alpha\left(e^{i t}\right) d t .
$$

Let us apply the linearity of the integral operator (4.6). Namely, denote by $\chi$ the characteristic function of an $\operatorname{arc} \gamma_{*}$ of $\partial \mathbf{D}$ where $\alpha$ is of bounded variation from the definition of $\mathcal{C B} \mathcal{V}$. Setting $\alpha_{*}=\alpha \chi$ and $\alpha_{0}=\alpha-\alpha_{*}$, we have that $\alpha=\alpha_{*}+\alpha_{0}$. Then $v=v_{*}+v_{0}$ where $v_{*}$ and $v_{0}$ correspond to $\alpha_{*}$ and $\alpha_{0}$ by formula (4.6). By the first item of the proof, there exists the angular $\operatorname{limit}_{\lim _{z \rightarrow \zeta}} v_{*}(z)=\beta_{*}(\zeta)$ q.e. on $\partial \mathbf{D}$ where $\beta_{*}: \partial \mathbf{D} \rightarrow \mathbf{R}$ is a measurable function with respect to the logarithmic capacity. Moreover, it is evident from formula (4.6) that $v_{0}(z) \rightarrow \beta_{0}(\zeta)$ as $z \rightarrow \zeta$ for all $\zeta \in \gamma_{*}$ 
where $\beta_{0}: \gamma_{*} \rightarrow \mathbf{R}$ is continuous on $\gamma_{*}$. Thus, setting $\beta=\beta_{*}+\beta_{0}$ on $\gamma_{*}$, we obtain the conclusion of Lemma 4.1, because the collection of such arcs $\gamma_{*}$ is countable and the completion of this collection on $\partial \mathbf{D}$ has zero logarithmic capacity.

\section{The Hilbert problem for analytic functions in the disk}

Now we are ready to give a solution to the Hilbert boundary value problem for analytic functions in the unit disk, assuming that the coefficient $\lambda$ is of countable bounded variation and the boundary date $\varphi$ is measurable with respect to the logarithmic capacity.

Theorem 5.1. Let $\lambda: \partial \mathbf{D} \rightarrow \partial \mathbf{D}$ be in the class $\mathcal{C B V}(\partial \mathbf{D})$ and $\varphi: \partial \mathbf{D} \rightarrow \mathbf{R}$ be measurable with respect to the logarithmic capacity. Then there is an analytic function $f: \mathbf{D} \rightarrow \mathbf{C}$ that has the angular limit

$$
\lim _{z \rightarrow \zeta} \operatorname{Re}[\overline{\lambda(\zeta)} f(z)]=\varphi(\zeta) \quad \text { q.e. on } \partial \mathbf{D} \text {. }
$$

Proof. By Proposition 2.5, the function $\alpha_{\lambda} \in L_{c}^{\infty}(\partial \mathbf{D}) \cap \mathcal{C B V}(\partial \mathbf{D})$. Therefore

$$
g(z):=\frac{1}{2 \pi i} \int_{\partial \mathbf{D}} \alpha_{\lambda}(\zeta) \frac{z+\zeta}{z-\zeta} \frac{d \zeta}{\zeta}, \quad z \in \mathbf{D}
$$

is analytic function with $u(z)=\operatorname{Re} g(z) \rightarrow \alpha_{\lambda}(\zeta)$ as $z \rightarrow \zeta$ for every $\zeta \in \partial \mathbf{D}$ except a set of the discontinuity points for the function $\alpha_{\lambda}$, which has zero logarithmic capacity, see e.g. [25, Corollary IX.1.1] and [34, Theorem I.D.2.2]. Note that the function $\mathcal{A}(z):=\exp \{i g(z)\}$ is also analytic.

By Lemma 4.1 there is a function $\beta: \partial \mathbf{D} \rightarrow \mathbf{R}$ that has the angular limit $v(z)=$ $\operatorname{Im} g(z) \rightarrow \beta(\zeta)$ as $z \rightarrow \zeta$ q.e. on $\partial \mathbf{D}$ and $\beta$ is measurable with respect to the logarithmic capacity. Thus, by Corollary 4.1 in [18] there exists an analytic function $\mathcal{B}: \mathbf{D} \rightarrow \mathbf{C}$ that has the angular limit $U(z)=\operatorname{Re} \mathcal{B}(z) \rightarrow \varphi(\zeta) \exp \{\beta(\zeta)\}$ as $z \rightarrow \zeta$ q.e. on $\partial \mathbf{D}$. Finally, an elementary computation shows that the desired function has the form $f=\mathcal{A B}$.

\section{The Hilbert problem for the Beltrami equation}

We say that a function $f: D \rightarrow \mathbf{C}$ is a regular solution of the Beltrami equation (1.2) if $f$ is continuous, discrete and open, has the first generalized derivatives and satisfies (1.2) a.e. in $D$. We also say that $f$ is a regular solution of the Hilbert boundary value problem (1.1) for the Beltrami equation (1.2) if $f$ in addition satisfies (1.1) q.e. on $\partial D$ along nontangential paths in $D$.

Recall that a mapping $f: D \rightarrow \mathbf{C}$ is called discrete if the pre-image $f^{-1}(z)$ consists of isolated points for every $z \in \mathbf{C}$, and open if $f$ maps every open set $U \subseteq D$ onto an open set in C. By the known Stoïlow result, see e.g. [53], every regular solution $f$ of (1.2) has the representation $f=h \circ g$ where $g$ is a homeomorphic solution of (1.2) and $h$ is an analytic function.

Theorem 6.1. Let $D$ be a Jordan domain with the quasihyperbolic boundary condition and let $\partial D$ have a tangent q.e. Suppose that $\mu: D \rightarrow \mathbf{C}$ is in $L^{\infty}(D)$ with $\|\mu\|_{\infty}<1, \lambda: \partial D \rightarrow \mathbf{C},|\lambda(\zeta)| \equiv 1$, is in $\mathcal{C B V}(\partial D)$ and $\varphi: \partial D \rightarrow \mathbf{R}$ is a measurable function with respect to the logarithmic capacity. Then the Hilbert problem (1.1) for the Beltrami equation (1.2) has a regular solution. 
Proof. Let $g$ be a conformal mapping of $D$ onto $\mathbf{D}$ that exists by the Riemann mapping theorem, see e.g. [25, Theorem II.2.1]. Setting in the unit disk D

$$
\nu(w):=\left(\mu \frac{g^{\prime}}{g^{\prime}}\right) \circ g^{-1}(w)
$$

we see that $\nu \in L^{\infty}(\mathbf{D})$ and $\|\nu\|_{\infty}=\|\mu\|_{\infty}<1$. Hence, by the Measurable Riemann Mapping theorem, see e.g. [3], [14] and [38], there is a quasiconformal mapping $G$ of D onto itself, $G(0)=0$, satisfying the Beltrami equation $G_{\bar{w}}=\nu(w) G_{w}$ a.e. in D.

By the reflection principle, see e.g. [38, Theorem I.8.4], $G$ can be extended to a quasiconformal mapping $\tilde{G}$ of $\mathbf{C}$ onto itself. Both functions $G_{*}:=\left.\tilde{G}\right|_{\partial \mathbf{D}}$ and $G_{*}^{-1}$ are Hölder continuous, see [13, Theorem 3.5], and also [38, Theorem II.4.3].

Now, by the Carathéodory theorem, see e.g. [25, Theorem II.3.4], $g$ is extended to a homeomorphism $\tilde{g}$ of $\bar{D}$ onto $\overline{\mathbf{D}}$. By [9, Corollary of Theorem 1], $g_{*}:=\left.\tilde{g}\right|_{\partial D}$ and its inverse function are Hölder continuous.

Thus, the mapping $h_{*}:=G_{*} \circ g_{*}: \partial D \rightarrow \partial \mathbf{D}$ and its inverse are also Hölder continuous. In particular, then $\Lambda:=\lambda \circ h_{*}^{-1} \in \mathcal{C B V}(\partial \mathbf{D})$ and $\Phi:=\varphi \circ h_{*}^{-1}$ is measurable with respect to logarithmic capacity by Remarks 2.1 and 2.6 .

Next, by Theorem 5.1 there is an analytic function $\mathcal{A}: \mathbf{D} \rightarrow \mathbf{C}$ that has the angular limit

$$
\lim _{\omega \rightarrow \eta} \operatorname{Re}\{\overline{\Lambda(\eta)} \mathcal{A}(\omega)\}=\Phi(\eta) \quad \text { q.e. on } \partial \mathbf{D} \text {. }
$$

Setting $h:=G \circ g$, we see, by an elementary computation, see e.g. [3, (1.C.1)], that $h_{z}=G_{w} \circ g(z) g^{\prime}(z)$ and $h_{\bar{z}}=G_{\bar{w}} \circ g(z) \overline{g^{\prime}(z)}$ a.e. in $D$, i.e. $h$ is a quasiconformal mapping of $D$ onto $\mathbf{D}$ satisfying equation (1.2) a.e. in $D$.

Let us consider the function $f:=\mathcal{A} \circ h$. Since $f_{z}=\mathcal{A}^{\prime} \circ h(z) h_{z}$ and $f_{\bar{z}}=$ $\mathcal{A}^{\prime} \circ h(z) h_{\bar{z}}$ a.e. in $D$, we see that $f$ satisfies the equation (1.2). On the other hand, the mapping $f$ is continuous, open and discrete, and therefore $f$ is the regular solution of (1.2). It remains to show that $f$ satisfies also the boundary condition (1.1).

Indeed, by the Lindelöf theorem, see e.g. [34, Theorem II.C.2], if $\partial D$ has a tangent at a point $\zeta$, then $\arg [g(\zeta)-g(z)]-\arg [\zeta-z] \rightarrow$ const as $z \rightarrow \zeta$. In other words, the images under the conformal mapping $g$ of sectors in $D$ with a vertex at $\zeta$ is asymptotically the same as sectors in $\mathbf{D}$ with a vertex at $w=g(\zeta)$. Consequently, nontangential paths in $D$ are transformed under $g$ into nontangential paths in $\mathbf{D}$ and inversely q.e. on $\partial D$ and $\partial \mathbf{D}$, respectively, because $D$ is almost smooth and $g_{*}$ and $g_{*}^{-1}$ keep sets of logarithmic capacity zero.

Moreover, it is known that the distortion of angles under a quasiconformal mapping is bounded, see e.g. [1, 2, 44]. Hence the mapping $\tilde{G}: \mathbf{C} \rightarrow \mathbf{C}$ and its inverse also transform nontangential paths into nontangential paths and $G_{*}$ and $G_{*}^{-1}$ keep sets of logarithmic capacity zero. Consequently, $h: D \rightarrow \mathbf{D}$ and $h^{-1}: \mathbf{D} \rightarrow D$ also transform nontangential paths into nontangential paths q.e. on $\partial D$ and $\partial \mathbf{D}$, respectively. Thus, (6.2) implies the existence of the angular limit (1.1) q.e. on $\partial D$.

Remark 6.2. The regular solution $f$ of the Hilbert boundary value problem for the Beltrami equation given in Theorem 6.1 has the following representation $f=\mathcal{A} \circ G \circ g$. Here $g: D \rightarrow \mathbf{D}$ stands for a conformal mapping, $G: \mathbf{D} \rightarrow \mathbf{D}$ is a quasiconformal mapping, normalized by $G(0)=0$ and satisfying the Beltrami equation with the coefficient $\nu$ in (6.1). Finally, $\mathcal{A}: \mathbf{D} \rightarrow \mathbf{C}$ is the analytic solution of the Hilbert problem with coefficient $\Lambda=\lambda \circ h_{*}^{-1}$ and boundary data $\Phi=\varphi \circ h_{*}^{-1}$, 
where $h=G \circ g$ and $h_{*}$ is the corresponding boundary homeomorphism of $\partial D$ onto $\partial \mathbf{D}$.

\section{On Dirichlet, Neumann and Poincare problems}

We reduce these boundary value problems to suitable Hilbert problems studied above and start with the Laplace equation. In particular, choosing $\mu \equiv 0$ and $\lambda \equiv 1$ in Theorem 6.1, we immediately obtain the following solution of the Dirichlet boundary value problem.

Corollary 7.1. Let $D$ be a Jordan domain with the quasihyperbolic boundary condition and let $\partial D$ have a tangent q.e. Suppose $\varphi: \partial D \rightarrow \mathbf{R}$ is measurable with respect to the logarithmic capacity. Then there exists a harmonic function $u: D \rightarrow \mathbf{C}$ that has the angular limit

$$
\lim _{z \rightarrow \zeta} u(z)=\varphi(\zeta) \quad \text { q.e. on } \partial D \text {. }
$$

We proceed to the study of nonclassical solutions of the Neumann boundary value problem. For this goal, we will study the more general problem on directional derivatives, that in turn is a partial case of the Poincare boundary value problem.

First of all, let us recall the classical setting of the problem on directional derivatives for the Laplace equation in the unit disk $\mathbf{D}$ : To find a twice continuously differentiable function $u: \mathbf{D} \rightarrow \mathbf{R}$ that admits a continuous extension to the boundary $\partial \mathbf{D}$ together with its first partial derivatives, satisfies the Laplace equation

$$
\Delta u:=\frac{\partial^{2} u}{\partial x^{2}}+\frac{\partial^{2} u}{\partial y^{2}}=0 \quad \forall z \in \mathbf{D}
$$

and the boundary condition

$$
\frac{\partial u}{\partial \nu}=\varphi(\zeta) \quad \forall \zeta \in \partial \mathbf{D}
$$

Here $\varphi: \partial \mathbf{D} \rightarrow \mathbf{R}$ stands for a prescribed continuous function and $\frac{\partial u}{\partial \nu}$ denotes the derivative of $u$ at the point $\zeta$ in the direction $\nu=\nu(\zeta),|\nu(\zeta)|=1$, i.e.,

$$
\frac{\partial u}{\partial \nu}:=\lim _{t \rightarrow 0} \frac{u(\zeta+t \nu)-u(\zeta)}{t} .
$$

The Neumann boundary value problem for the Laplace equation is a special case of the above problem with the following boundary condition

$$
\frac{\partial u}{\partial n}=\varphi(\zeta) \quad \forall \zeta \in \partial \mathbf{D},
$$

where $n$ denotes the unit interior normal to $\partial \mathbf{D}$ at the point $\zeta$.

Let us note that the above problem on directional derivatives is a partial case of the Poincare boundary value problem

$$
a u+b \frac{\partial u}{\partial \nu}=\varphi(\zeta) \quad \forall \zeta \in \partial \mathbf{D}
$$

where $a=a(\zeta)$ and $b=b(\zeta)$ are real-valued functions given on $\partial \mathbf{D}$.

It is well known, that the Neumann problem, in general, has no classical solution. The necessary condition for the solvability is that the integral of the function $\varphi$ over $\partial \mathbf{D}$ is equal zero, see e.g. [40]. Recently, it was established the existence of nonclassical solutions of the Neumann problem for the Laplace equation in rectifiable Jordan domains for arbitrary measurable data with respect to the natural parameter, see [49]. Then the results have been extended to linear divergence equations in 
Lipschitz domains with arbitrary measurable data with respect to the logarithmic capacity, see [57]. Here we extend the corresponding results to wider classes of domains and boundary functions.

Theorem 7.2. Let $D$ be a Jordan domain with the quasihyperbolic boundary condition and let $\partial D$ have a tangent q.e. Suppose that $\nu: \partial D \rightarrow \mathbf{C},|\nu(\zeta)| \equiv 1$, is in the class $\mathcal{C B V}$ and $\varphi: \partial D \rightarrow \mathbf{R}$ is measurable with respect to the logarithmic capacity. Then there exists a harmonic function $u: D \rightarrow \mathbf{R}$ that has the angular limit

$$
\lim _{z \rightarrow \zeta} \frac{\partial u}{\partial \nu}=\varphi(\zeta) \quad \text { q.e. on } \partial D
$$

Proof. Indeed, by Theorem 6.1 there exists an analytic function $f: D \rightarrow \mathbf{C}$ that has the angular limit

$$
\lim _{z \rightarrow \zeta} \operatorname{Re}[\nu(\zeta) f(z)]=\varphi(\zeta)
$$

q.e. on $\partial D$. Note that an indefinite integral $F$ of $f$ in $D$ is also an analytic function and, correspondingly, the harmonic functions $u=\operatorname{Re} F$ and $v=\operatorname{Im} F$ satisfy the Cauchy-Riemann system $v_{x}=-u_{y}$ è $v_{y}=u_{x}$. Hence

$$
f=F^{\prime}=F_{x}=u_{x}+i v_{x}=u_{x}-i u_{y}=\overline{\nabla u}
$$

where $\nabla u=u_{x}+i u_{y}$ is the gradient of the function $u$ in the complex form. Thus, (7.7) follows from (7.8), i.e. $u$ is the desired harmonic function, because its directional derivative

$$
\frac{\partial u}{\partial \nu}=\operatorname{Re} \bar{\nu} \nabla u=\operatorname{Re} \nu \overline{\nabla u}=\langle\nu, \nabla u\rangle
$$

is the scalar product of $\nu$ and the gradient $\nabla u$.

Remark 7.3. We are able to say more in the case $\operatorname{Re}[n \bar{\nu}]>0$ where $n=n(\zeta)$ is the unit interior normal at the point $\zeta \in \partial D$. In view of $(7.7)$, since the limit $\varphi(\zeta)$ is finite, there is a finite limit $u(\zeta)$ of $u(z)$ as $z \rightarrow \zeta$ in $D$ along the straight line passing through the point $\zeta$ and being parallel to the vector $\nu(\zeta)$. Indeed, along this line, for $z$ and $z_{0}$ that are close enough to $\zeta$,

$$
u(z)=u\left(z_{0}\right)-\int_{0}^{1} \frac{\partial u}{\partial \nu}\left(z_{0}+\tau\left(z-z_{0}\right)\right) d \tau .
$$

Thus, at each point with the condition (7.7), there is the directional derivative

$$
\frac{\partial u}{\partial \nu}(\zeta):=\lim _{t \rightarrow 0} \frac{u(\zeta+t \nu)-u(\zeta)}{t}=\varphi(\zeta) .
$$

In particular, $\operatorname{Re}[n \bar{\nu}]=1$ in the case of the Neumann problem and, thus, we arrive, by Theorem 7.2 and Remark 7.3, at the following result.

Corollary 7.4. Let $D$ be a Jordan domain in $\mathbf{C}$ with the quasihyperbolic boundary condition and let the unit interior normal $n(\zeta)$ to the boundary $\partial D$ be in the class $\mathcal{C B V}$. Suppose that $\varphi: \partial D \rightarrow \mathbf{R}$ is measurable with respect to the logarithmic capacity. Then one can find a harmonic function $u: D \rightarrow \mathbf{C}$ such that q.e. on $\partial D$ there exist:

1) the finite limit along the normal $n(\zeta)$

$$
u(\zeta):=\lim _{z \rightarrow \zeta} u(z)
$$


2) the normal derivative

$$
\frac{\partial u}{\partial n}(\zeta):=\lim _{t \rightarrow 0} \frac{u(\zeta+t n)-u(\zeta)}{t}=\varphi(\zeta),
$$

3) the angular limit

$$
\lim _{z \rightarrow \zeta} \frac{\partial u}{\partial n}(z)=\frac{\partial u}{\partial n}(\zeta)
$$

Recall that, see e.g. [6, Theorem 16.1.6], if $f=u+i v$ is a regular solution of the Beltrami equation (1.2), then the function $u$ is a continuous generalized solution of the divergence type equation

$$
\operatorname{div} A(z) \nabla u=0,
$$

called A-harmonic function, see [29], i.e. $u \in C \cap W_{\mathrm{loc}}^{1,1}(D)$ and

$$
\int_{D}\langle A(z) \nabla u, \nabla \varphi\rangle=0 \quad \forall \varphi \in C_{0}^{\infty}(D),
$$

where $A(z)$ is the matrix function:

$$
A=\left(\begin{array}{cc}
\frac{|1-\mu|^{2}}{1-|\mu|^{2}} & \frac{-2 \operatorname{Im} \mu}{1-|\mu|^{2}} \\
\frac{-2 \operatorname{Im} \mu}{1-|\mu|^{2}} & \frac{|1+\mu|^{2}}{1-|\mu|^{2}}
\end{array}\right) .
$$

As we see, the matrix function $A(z)$ in (7.10) is symmetric and its entries $a_{i j}=a_{i j}(z)$ are dominated by the quantity

$$
K_{\mu}(z)=\frac{1+|\mu(z)|}{1-|\mu(z)|}
$$

and, thus, they are bounded if Beltrami's equation (1.2) is not degenerate.

Vice verse, uniformly elliptic equations (7.9) with symmetric $A(z)$ and $\operatorname{det} A(z) \equiv$ 1 just correspond to nondegenerate Beltrami equations (1.2) with coefficient

$$
\mu=\frac{1}{\operatorname{det}(I+A)}\left(a_{22}-a_{11}-2 i a_{21}\right)=\frac{a_{22}-a_{11}-2 i a_{21}}{1+\operatorname{Tr} A+\operatorname{det} A} .
$$

Following [28], we denote by $\mathcal{B}$ the collection of all such matrix functions $A(z)$. Recall that the equation (7.9) is said to be uniformly elliptic, if $a_{i j} \in L^{\infty}$ and $\langle A(z) \eta, \eta\rangle \geq$ $\varepsilon|\eta|^{2}$ for some $\varepsilon>0$ and for all $\eta \in \mathbf{R}^{2}$.

Corollary 7.5. Let $D$ be a domain with the quasihyperbolic boundary condition and let $\partial D$ have a tangent q.e. Suppose that $A \in \mathcal{B}$ and $\varphi: \partial D \rightarrow \mathbf{R}$ is measurable with respect to logarithmic capacity. Then there exists $A$-harmonic function $u: D \rightarrow$ $\mathbf{R}$ satisfying the Dirichlet boundary condition (7.1).

Theorem 7.6. Let $D$ be a domain in $\mathbf{C}$ with the quasihyperbolic boundary condition and let $\partial D$ have a tangent q.e. Suppose that $A(z), z \in D$, is a matrix function in the class $\mathcal{B} \cap C^{\alpha}, \alpha \in(0,1), \nu: \partial D \rightarrow \mathbf{C},|\nu(\zeta)| \equiv 1$, is in the class $\mathcal{C B V}$ and $\varphi: \partial D \rightarrow \mathbf{R}$ is measurable with respect to logarithmic capacity. Then there exists $A$-harmonic function $u: D \rightarrow \mathbf{R}$ in the class $C^{1+\alpha}$ that has the angular limit

$$
\lim _{z \rightarrow \zeta} \frac{\partial u}{\partial \nu}(z)=\varphi(\zeta) \quad \text { q.e. on } \partial D \text {. }
$$

Proof. By the above remarks, a desired function $u$ is a real part of a solution $f$ in class $W_{\text {loc }}^{1,1}$ for the Beltrami equation (1.2) with $\mu \in C^{\alpha}$ given by the formula (7.11). By Lemma 1 in [28] $\mu$ is extended to a Hölder continuous function $\mu_{*}: \mathbf{C} \rightarrow \mathbf{C}$ of the class $C^{\alpha}$. Set $k=\max |\mu(z)|<1$ in $D$. Then, for every $k_{*} \in(k, 1)$, there is an open 
neighborhood $U$ of $D$ where $\left|\mu_{*}(z)\right| \leq k_{*}$. Let $D_{*}$ be a connected component of $U$ containing $\bar{D}$.

By the Measurable Riemann Mapping Theorem, see e.g. [3], [14] and [38], there is a quasiconformal mapping $h: D_{*} \rightarrow \mathbf{C}$ a.e. satisfying the Beltrami equation (1.2) with the complex coefficient $\mu^{*}:=\left.\mu_{*}\right|_{D_{*}}$ in $D_{*}$. Note that the mapping $h$ has the Hölder continuous first partial derivatives in $D_{*}$ with the same order of the Hölder continuity as $\mu$, see e.g. [32] and also [33]. Moreover, its Jacobian

$$
J_{h}(z) \neq 0 \quad \forall z \in D_{*},
$$

see e.g. Theorem V.7.1 in [38]. Thus, the directional derivative

$$
h_{\omega}(z)=\frac{\partial h}{\partial \omega}(z):=\lim _{t \rightarrow 0} \frac{h(z+t \omega)-h(z)}{t} \neq 0 \quad \forall z \in D_{*} \forall \omega \in \partial \mathbf{D}
$$

and it is continuous by the collection of the variables $\omega \in \partial \mathbf{D}$ and $z \in D_{*}$. Thus, the functions

$$
\nu_{*}(\zeta):=\frac{\left|h_{\nu(\zeta)}(\zeta)\right|}{h_{\nu(\zeta)}(\zeta)} \quad \text { and } \quad \varphi_{*}(\zeta):=\frac{\varphi(\zeta)}{\left|h_{\nu(\zeta)}(\zeta)\right|}
$$

are measurable with respect to the logarithmic capacity, see e.g. convergence arguments in [35], Section 17.1.

The logarithmic capacity of a set coincides with its transfinite diameter, see e.g. [20] and the point 110 in [42]. Moreover, quasiconformal mappings are Hölder continuous on compacta, see e.g. [38, Theorem II.4.3]. Hence the mappings $h$ and $h^{-1}$ transform sets of logarithmic capacity zero on $\partial D$ into sets of logarithmic capacity zero on $\partial D^{*}$, where $D^{*}:=h(D)$, and vice versa.

Further, the functions $\mathcal{N}:=\left.\nu_{*} \circ h^{-1}\right|_{\partial D^{*}}$ and $\Phi:=\left.\left(\varphi_{*} / h_{\nu}\right) \circ h^{-1}\right|_{\partial D^{*}}$ are measurable with respect to the logarithmic capacity. Indeed, a measurable set with respect to the logarithmic capacity is transformed under the mappings $h$ and $h^{-1}$ into measurable sets with respect to the logarithmic capacity. Really, such a set can be represented as the union of a sigma-compactum and a set of logarithmic capacity zero. On the other hand, the compacta are transformed under continuous mappings into compacta and the compacta are measurable with respect to the logarithmic capacity.

Recall that the distortion of angles under quasiconformal mappings $h$ è $h^{-1}$ is bounded, see e.g. [1, 2, 44]. Thus, nontangential paths to $\partial D$ are transformed into nontangential paths to $\partial D^{*}$ for a.e. $\zeta \in \partial D$ with respect to the logarithmic capacity and inversely.

By Theorem 7.2, one can find a harmonic function $U: D^{*} \rightarrow \mathbf{R}$ that has the angular limit

$$
\lim _{w \rightarrow \xi} \frac{\partial U}{\partial \mathcal{N}}(w)=\Phi(\xi) \quad \text { q.e. on } \partial D^{*}
$$

Moreover, one can find a harmonic function $V$ in the simply connected domain $D^{*}$ such that $F=U+i V$ is an analytic function and, thus, $u:=\operatorname{Re} f=U \circ h$, where $f:=F \circ h$, is a desired $A$-harmonic function in Theorem 7.6 because $f$ is a regular solution of the corresponding Beltrami equation (1.2) and also

$$
\begin{aligned}
u_{\nu} & =\left\langle\nabla U \circ h, h_{\nu}\right\rangle=\left\langle\nu_{*} \nabla U \circ h, \nu_{*} h_{\nu}\right\rangle \\
& =\left\langle\frac{\partial U}{\partial \mathcal{N}} \circ h, \nu_{*} h_{\nu}\right\rangle=\frac{\partial U}{\partial \mathcal{N}} \circ h \operatorname{Re}\left(\nu_{*} h_{\nu}\right) .
\end{aligned}
$$


The following statement concerning to the Neumann problem for $A$-harmonic functions is a partial case of Theorem 7.6.

Corollary 7.7. Let $D$ be a domain in $\mathbf{C}$ with the quasihyperbolic boundary condition and let $\partial D$ have a tangent q.e. Suppose that $A(z), z \in D$, is a matrix function in the class $\mathcal{B} \cap C^{\alpha}, \alpha \in(0,1)$, the interior unit normal $n=n(\zeta)$ to $\partial D$ is in the class $\mathcal{C B V}$ and $\varphi: \partial D \rightarrow \mathbf{R}$ is measurable with respect to the logarithmic capacity. Then there is an A-harmonic function $u: D \rightarrow \mathbf{R}$ of the class $C^{1+\alpha}$ such that q.e. on $\partial D$ there exist:

1) the finite limit along the normal $n(\zeta)$,

$$
u(\zeta):=\lim _{z \rightarrow \zeta} u(z)
$$

2) the normal derivative

$$
\frac{\partial u}{\partial n}(\zeta):=\lim _{t \rightarrow 0} \frac{u(\zeta+t n)-u(\zeta)}{t}=\varphi(\zeta)
$$

3) the angular limit

$$
\lim _{z \rightarrow \zeta} \frac{\partial u}{\partial n}(z)=\frac{\partial u}{\partial n}(\zeta)
$$

\section{On the dimension of the spaces of solutions}

It was established in [18], Theorem 8.1, that the space of all harmonic functions $u: \mathbf{D} \rightarrow \mathbf{R}$ that has the angular limit $\lim _{z \rightarrow \zeta} u(z)=0$ q.e. on $\partial \mathbf{D}$ has the infinite dimension. This statement can be extended to the Hilbert boundary value problem because we reduced this problem in Theorem 5.1 to the corresponding two Dirichlet problems.

Theorem 8.1. Let $\lambda: \partial \mathbf{D} \rightarrow \partial \mathbf{D}$ be in class $\mathcal{C B V}(\partial \mathbf{D})$ and $\varphi: \partial \mathbf{D} \rightarrow \mathbf{R}$ be measurable with respect to logarithmic capacity. Then the space of all analytic functions $f: \mathbf{D} \rightarrow \mathbf{C}$ with the angular limit

$$
\lim _{z \rightarrow \zeta} \operatorname{Re}\{\overline{\lambda(\zeta)} f(z)\}=\varphi(\zeta) \quad \text { q.e. on } \partial \mathbf{D}
$$

has the infinite dimension.

Proof. Let $u: \mathbf{D} \rightarrow \mathbf{R}$ be a harmonic function that has the angular limit 0 q.e. on $\partial \mathbf{D}$ from [18, Theorem 8.1]. Then there is the unique harmonic function $v: \mathbf{D} \rightarrow \mathbf{R}$ with $v(0)=0$, such that $\mathcal{C}=u+i v$ is an analytic function. Thus, setting in the proof of Theorem $5.1 \mathrm{~g}=\mathcal{A}(\mathcal{B}+\mathcal{C})$ instead of $f=\mathcal{A} \mathcal{B}$, we obtain by [18, Theorem 8.1] that the space of solutions of the Hilbert boundary value problem (8.1) for analytic functions in Theorem 5.1 has the infinite dimension.

Finally, since the proof of the rest of theorems and corollaries was sequentially reduced to Theorem 5.1, we come by Theorem 8.1 to the following conclusion.

Corollary 8.2. All the spaces of solutions of the boundary value problems in Theorems 6.1, 7.2, 7.6, Corollaries 7.1-7.7 also have the infinite dimension.

Recently it was established by us a number of effective criteria for the existence of solutions for the degenerate Beltrami equations, see e.g. [27]. That makes possible to consider the boundary value problems for such equations, too. However, the latter will demand a more deep study of properties of the mappings with finite distortion, see e.g. the monographs [30] and [39]. 
Acknowledgements. This work was partially supported by grants of Ministry of Education and Science of Ukraine, project number is 0119U100421. We would like also to thank our referee for useful remarks that made it to be possible improving the text.

\section{References}

[1] Agard, S.: Angles and quasiconformal mappings in space. - J. Anal. Math. 22, 1969, 177-200.

[2] Agard, S. B., and F. W. Gehring: Angles and quasiconformal mappings. - Proc. London Math. Soc. (3) 14a, 1965, 1-21.

[3] Ahlfors, L.: Lectures on quasiconformal mappings. - Van Nostrand, New York, 1966.

[4] Ahlfors L., and A. Beurling: The boundary correspondence under quasiconformal mappings. - Acta Math. 96, 1956, 125-142.

[5] Anderson G. D., M. K. Vamanamurthy, and M. K. Vuorinen: Conformal invariants, inequalities and quasiconformal maps. - Wiley-Intersci. Publ., New York, 1997.

[6] Astala, K., T. Iwaniec, and G. J. Martin: Elliptic differential equations and quasiconformal mappings in the plane. - Princeton Math. Ser. 48, Princeton Univ. Press, Princeton, 2009 .

[7] Astala, K., and P. Koskela: Quasiconformal mappings and global integrability of the derivative. - J. Anal. Math. 57, 1991, 203-220.

[8] Bari, N. K.: Trigonometric series. - Gos. Izd. Fiz.-Mat. Lit., Moscow, 1961 (in Russian); English transl.: A treatise on trigonometric series, Volumes I and II. - Macmillan Co., New York, 1964.

[9] Becker, J., and Ch. Pommerenke: Hölder continuity of conformal mappings and nonquasiconformal Jordan curves. - Comment. Math. Helv. 57:2, 1982, 221-225.

[10] Begenr, H.: Complex analytic methods for partial differential equations. An introductory text. -World Scientific Publishing Co., Inc., River Edge, NJ, 1994.

[11] Begehr, H., and G. Ch. Wen: Nonlinear elliptic boundary value problems and their applications. - Pitman Monographs and Surveys in Pure and Applied Mathematics 80, Longman, Harlow, 1996.

[12] Bojarski, B. V.: Homeomorphic solutions of Beltrami systems. - Dokl. Akad. Nauk SSSR (N.S.) 102, 1955, 661-664 (in Russian).

[13] Bojarski, B. V.: Generalized solutions of a system of differential equations of the first order and elliptic type with discontinuous coefficients. - Report Dept. Math. Stat. 118, Univ. of Jyväskylä, Jyväskylä, 2009; transl. from Mat. Sb. (N.S.) 43(85), 1957, 451-503.

[14] Bojarski, B., V. Gutlyanskit, O. Martio, and V. Ryazanov: Infinitesimal geometry of quasiconformal and bi-lipschitz mappings in the plane. - EMS Tracts in Math. 19, Eur. Math. Soc., Zürich, 2013.

[15] Carleson, L.: Selected problems on exceptional sets. - Van Nostrand Co., Inc., Princeton, 1971.

[16] Dovgoshey, O., O. Martio, V. Ryazanov, and M. Vuorinen: The Cantor function. Expo. Math. 24:1, 2006, 1-37.

[17] Duren, P.L.: Theory of $H^{p}$ spaces. - Pure Appl. Math. 38, Academic Press, New YorkLondon, 1970.

[18] Efimushin, A., and V. Ryazanov: On the Riemann-Hilbert problem for the Beltrami equations. - In: Complex analysis and dynamical systems VI. Part 2, Contemp. Math. 667, Israel Math. Conf. Proc., Amer. Math. Soc., Providence, RI, 2016, 299-316.

[19] Federer, H.: Geometric measure theory. - Springer-Verlag, Berlin, 1969. 
[20] FÉKete, M.: Über die Verteilung der Wurzeln bei gewissen algebraischen Gleichungen mit ganzzahligen Koeffizienten. - Math. Z. 17, 1923, 228-249.

[21] Gakhov, F. D.: Boundary value problems. - Dover Publications, Inc., New York, 1990.

[22] Gehring, F. W., and O. Martio: Quasiextremal distance domains and extension of quasiconformal mappings. - J. Analyse Math. 45, 1985, 181-206.

[23] Gehring, F. W., and O. Martio: Lipschitz classes and quasiconformal mappings. - Ann. Acad. Sci. Fenn. Ser. A I Math. 10, 1985, 203-219.

[24] Gehring, F. W., and B. P PALKA: Quasiconformally homogeneous domains. - J. Analyse Math. 30, 1976, 172-199.

[25] Goluzin, G. M.: Geometric theory of functions of a complex variable. - Transl. Math. Monogr. 26, Amer. Math. Soc., Providence, R.I., 1969.

[26] Gutlyanskit, V., and V. Ryazanov: On recent advances in boundary value problems in the plane. - Ukr. Mat. Visn. 13:2, 2016, 167-212.

[27] Gutlyanskit, V., V. Ryazanov, U. Srebro, and E. Yakubov: The Beltrami equation: A geometric approach. - Dev. Math. 26, Springer, New York, 2012.

[28] Gutlyanskit, V., V. Ryazanov, and A. Yefimushkin: On the boundary value problems for quasiconformal functions in the plane. - Ukr. Mat. Visn. 12:3, 2015, 363-389; English transl. J. Math. Sci. (N.Y.) 214:2, 2016, 200-219.

[29] Heinonen, J., T. Kilpeläinen, and O. Martio: Nonlinear potential theory of degenerate elliptic equations. - Oxford Math. Monogr., Oxford Science Publications, The Clarendon Press, Oxford Univ. Press, New York, 1993.

[30] Hencl, S., and P. Koskela: Lectures on mappings of finite distortion. - Lecture Notes in Math. 2096, Springer, Cham, 2014.

[31] Hilbert, D.: Über eine Anwendung der Integralgleichungen auf eine Problem der Funktionentheorie. - Verhandl. des III Int. Math. Kongr., Heidelberg, 1904.

[32] IWANiEC, T.: Regularity of solutions of certain degenerate elliptic systems of equations that realize quasiconformal mappings in $n$-dimensional space. - In: Differential and integral equations. Boundary value problems, Tbilis. Gos. Univ., Tbilisi, 1979, 97-111.

[33] IwANiec, T.: Regularity theorems for solutions of partial differential equations for quasiconformal mappings in several dimensions. - Dissertationes Math. (Rozprawy Mat.) 198, 1982, $1-45$.

[34] Koosis, P.: Introduction to $H_{p}$ spaces. - Cambridge Tracts in Math. 115, Cambridge Univ. Press, Cambridge, 1998.

[35] Krasnosel'skit, M. A., P. P. Zabreiko, E. I. Pustyl'nik, and P. E. Sobolevskii: Integral operators in spaces of summable functions. - Monographs and Textbooks on Mechanics of Solids and Fluids, Mechanics: Analysis, Noordhoff International Publishing, Leiden, 1976.

[36] Ladyzhenskaya, O. A., and N. N. Ural'tseva: Linear and quasilinear elliptic equations. Academic Press, New York-London, 1968; English transl. from Lineinye i kvazilineinye uravneniya ellipticheskogo tipa, Nauka, Moscow, 1964.

[37] Landkof, N. S.: Foundations of modern potential theory. - Grundlehren Math. Wiss. 180, Springer-Verlag, New York-Heidelberg, 1972.

[38] Lehto, O., and K. J. Virtanen: Quasiconformal mappings in the plane. - Springer-Verlag, Berlin, Heidelberg, 1973.

[39] Martio, O., V. Ryazanov, U. Srebro, and E. Yakubov: Moduli in modern mapping theory. - Springer Monogr. Math., Springer, New York etc., 2009.

[40] Mikhlin, S. G.: Partielle Differentialgleichungen in der mathematischen Physik. - Math. Lehrbücher und Monographien 30, Akademie-Verlag, Berlin, 1978.

[41] Muskhelishvili, N. I.: Singular integral equations. Boundary problems of function theory and their application to mathematical physics. - Dover Publications, Inc., New York, 1992. 
[42] Nevanlinna, R.: Eindeutige analytische Funktionen. - Ann Arbor, Michigan, 1944.

[43] Noshiro, K.: Cluster sets. - Springer-Verlag, Berlin etc., 1960.

[44] TAari, O.: Charakterisierung der Quasikonformität mit Hilfe der Winkelverzerrung. - Ann. Acad. Sci. Fenn. Ser. A I Math. 390, 1966, 1-43 (in German).

[45] Trogdon, Th., and Sh. Olver: Riemann-Hilbert problems, their numerical solution, and the computation of nonlinear special functions. - Society for Industrial and Applied Mathematics (SIAM), Philadelphia, 2016.

[46] Pommerenke, Ch.: Boundary behaviour of conformal maps. - Grundlehren Math. Wiss. 299, Springer-Verlag, Berlin, 1992.

[47] Ryazanov, V. I.: On the Riemann-Hilbert problem without index. - Ann. Univ. Bucharest Ser. Math. 5:1 (LXIII), 2014, 169-178.

[48] Ryazanov, V.: Infinite dimension of solutions of the Dirichlet problem. - Open Math. 13, $2015,348-350$.

[49] Ryazanov, V.: On Neumann and Poincare problems for Laplace equation. - Analysis and Mathematical Physics 7:3, 2017, 285-289.

[50] RYAzAnov, V.: On the boundary behavior of conjugate harmonic functions. - Proceedings of Inst. Appl. Math. Mech. of Nat. Acad. Sci. of Ukraine 31, 2017, 117-123; see also arXiv.org: $1710.00323 \mathrm{v} 3$ [math.CV].

[51] Ryazanov, V.: The Stieltjes integrals in the theory of harmonic functions. - Zap. Nauchn. Sem. S.-Peterburg. Otdel. Mat. Inst. Steklova (POMI) 467, 2018; Issledovaniya po Lineinym Operatoram i Teorii Funktsii 46, 2018, 151-168; English transl. in J. Math. Sci. (N.Y.) 243:6, 2019, 922-933.

[52] Saks, S.: Theory of the integral. - Dover Publications Inc., New York, 1964.

[53] SтоÏLOW, S.: Lektsii o topologicheskikh printsipakh teorii analiticheskikh funktsii. - Nauka, Moscow, 1964 (in Russian).

[54] Twomey, J. B.: Tangential boundary behaviour of the Cauchy integral. - J. London Math. Soc. (2) $37: 3,1988,447-454$.

[55] Vekua, I. N.: Generalized analytic functions. - Pergamon Press, London etc., 1962.

[56] Vuorinen, M.: Conformal geometry and quasiregular mappings. - Lecture Notes in Math. 1319, Springer-Verlag, Berlin, 1988.

[57] Yefimushkin A.: On Neumann and Poincare problems in $A$-harmonic analysis. -Advances in Analysis 1:2, 2016, 114-120.

Received 29 October 2018 • Accepted 11 October 2019 